\section{Reporting data on long-term follow-up of critical care trials}

\author{
May Hua, ${ }^{1}$ Hannah Wunsch ${ }^{1,2,3}$
}

Over the past 20 years, the lens of critical care research has widened, with 60 or 90 days becoming an increasingly common end point for observation of mortality and other outcomes. ${ }^{1-6}$ There have also been an increase in studies that follow patients for many years to understand how and when recovery occurs and whether risks attributable to critical illness may diminish. ${ }^{7-9}$ Correspondingly, when new therapies aimed at improving outcomes are studied, there is increasing recognition that reporting of short-term (ie, hospital) outcomes is not enough and that evaluation of the long-term effects of the intervention can be very important.

Dinglas et al present long-term follow-up data of patients enrolled in the Statins in Acutely Injured Lungs in Sepsis (SAILS) trial, which examined the use of rosuvastatin in patients with sepsis-associated acute respiratory distress syndrome (ARDS). ${ }^{10}$ The SAILS trial randomised patients within $48 \mathrm{~h}$ of enrolment to receive either rosuvastatin or placebo for a maximum of 28 days (or until the third day after discharge from the intensive care unit (ICU), hospital discharge or death). The primary outcome of the original study was 60-day in-hospital mortality. As part of the planned analysis, the authors evaluated the long-term outcome of these patients to determine the safety of in-hospital statin use in survivors of sepsis-associated ARDS. ${ }^{11}$ The rationale was that there are known adverse effects of statins on skeletal muscle and psychological symptoms that can occur, and critically ill patients represent a population already at risk of physical and psychological difficulties.

The authors collected detailed data on physical performance and psychological symptoms at 6 and 12 months, using multiple validated scales to measure

\footnotetext{
${ }^{1}$ Department of Anesthesiology, Columbia University, New York, New York, USA; ${ }^{2}$ Department of Critical Care Medicine, Sunnybrook Health Sciences Centre, Toronto, Ontario, Canada; ${ }^{3}$ Department of Anesthesia and Interdepartmental Division of Critical Care Medicine, University of Toronto, Toronto, Ontario, Canada

Correspondence to Dr Hannah Wunsch, Department of Critical Care Medicine, Sunnybrook Hospital, 2075 Bayview Ave, Rm D1.08, Toronto, ON M4N 3M5, Canada; Hannah.Wunsch@sunnybrook.ca
}

functional and mental health outcomes. They had minimal loss to follow-up, although it is notable that the performance-based physical outcome evaluations were done in a much smaller sample of patients. They found no difference in outcome between patients who did and patients who did not receive rosuvastatin and this effect was consistent across multiple predefined subgroups, suggesting that short-term statin usage in a critically ill population has an acceptable safety profile but was not associated with any improvements in outcome.

The original SAILS trial was stopped for futility after enrolment of 745 patients. The data in the current study would have been most salient had the SAILS trial been positive, as it would have elucidated whether observed differences were sustained, or (as in the original study of activated protein $\mathrm{C}$ ) if overall differences in outcomes were transient, with benefit only in a specific subgroup. ${ }^{12}$ The question of the safety of short-term rosuvastatin use would also have been more compelling in a positive trial, since the possibility of harm from a new treatment that may be widely adopted certainly warrants rigorous assessment. Finally, as this study did not collect data on continued statin use, these data only provide support for the safety of short-term statin use and do not shed light on whether adverse effects would be seen with continued administration in this select population of survivors.

With negative findings, these data primarily provide more information on the burden of morbidity and mortality for patients who have a specific type of critical illness (sepsis-associated ARDS). In this cohort, survivors had lower scores on both components of the SF-36 compared with population norms. Psychological symptoms were prevalent, with anxiety symptoms occurring most frequently, followed by depressive symptoms and those of post-traumatic stress disorder. Patients were also unlikely to return to work at 6 and 12 months and had decrements in functional performance with shorter distances on the 6 min walk test. Overall, these outcomes are similar to previously published estimates. 71314

One continued problem with studies of long-term outcome of critical illness is that they often lack robust data on pre-ICU morbidity, limiting our understanding of whether patients destined for poor psychological, cognitive and functional outcomes are more likely to have critical illness or whether an episode of critical illness plays a causative role in the development of subsequent morbidity. This study lacked pre-ICU information on existing psychological and functional deficits and therefore cannot untangle the issue of whether the observed outcome represent a pre-ICU burden versus new deficits. Ensuring adequate data on pre-ICU status in long-term outcome studies is a pressing concern now that we have moved away from measuring only death (a state everyone is 'negative' for prior to ICU admission) and towards more complex outcomes such as functional disability, frailty and cognitive dysfunction. In theory, availability of electronic health records that can be universally accessed should dramatically help with this problem, but this benefit remains theoretical in most healthcare systems.

As the importance of assessing longterm outcome is well established in critical illness, we are now faced with the question of when we should view the collection of long-term follow-up data as a compulsory step to delineate the implications of prescribing or implementing a new therapy to a large population of patients and when to acknowledge that such follow-up is unlikely to yield new information. Conducting long-term follow-up is costly and time-consuming for researchers and impacts patients and their families. Some survivors of critical illness live with impairments and their caregivers can also suffer from psychological symptoms and fatigue ${ }^{15}$; we need to be cognisant of the potential additional burden placed on our patients and families by asking them to undergo multiple assessments.

In an era of limited funding, we must be confident that inclusion of long-term follow-up as part of a study protocol is necessary. Ideally, there should be a mechanistic rationale for why long-term follow-up is justified, particularly if the intervention is delivered in a limited time period and no signal for benefit or harm is seen in the short term. For example, long-term follow-up seems important in studies of interventions aimed at physical or psychological 'rehabilitation', where one might anticipate a delayed impact on patients. However, the long-term follow-up of all randomised controlled trials involving critically ill patients is not 
likely to be useful or particularly feasible. Of course, there may be unanticipated findings in some studies, but the potential for such a result needs to be weighed against the enormous costs of these assessments. This is easier to say than to do, as long-term follow-up often occurs in parallel with study enrolment and short-term assessment over multiple years. Therefore, the need for follow-up and its appropriate length will likely need to be carefully adjudicated prior to the start of any study.

Additionally, the current methodological heterogeneity in long-term outcome studies presents a large challenge. There is currently no consensus as to which outcome is most important to measure, which instrument should be used to measure a given outcome and whether that instrument remains valid regardless of the time point of measurement. Efforts are currently underway to establish standardised outcome measurements that will also facilitate comparison of outcomes across studies and potentially allow for easier pooling of data. ${ }^{16} 17$

The persistence of significant symptomatology months to years after critical illness illustrated in this study and others does underscore the need to better understand the underlying mechanisms and to identify interventions that may modify these outcomes in situations where there is a change from a patient's pre-ICU status. As we continue to treat increasingly large numbers of patients in the ICU with a corresponding increase in survivorship, ${ }^{18}$ it remains our responsibility to find ways to improve both short and longterm survival, to confirm that our attempts to do so do not cause further harm and to improve quality of life for patients who do survive. However, equally urgent is the need to be judicious in the use of our time, resources and good will of our patients and families by determining when long-term follow-up in studies is truly necessary.

Contributors $\mathrm{MH}$ and HW take full responsibility for the manuscript.

Competing interests None declared.

Provenance and peer review Commissioned; externally peer reviewed.

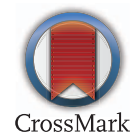

To cite Hua M, Wunsch H. Thorax 2016;71:395-396.

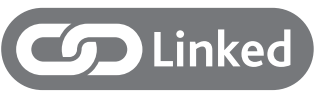

- http://dx.doi.org/10.1136/thoraxjnl-2015-208017

Thorax 2016;71:395-396.

doi:10.1136/thoraxjn-2016-208466

\section{REFERENCES}

1 Finfer S, Chittock DR, Su SY, et al., NICE-SUGAR Study Investigators. Intensive versus conventional glucose control in critically ill patients. N Engl I Med 2009;360:1283-97.

2 Peake SL, Delaney A, Bailey M, et al., ARISE Investigators, ANZICS Clinical Trials Group. Goal-directed resuscitation for patients with early septic shock. N Engl I Med 2014;371:1496-506.

3 Mouncey PR, Osborn TM, Power GS, et al. Trial of early, goal-directed resuscitation for septic shock. N Engl J Med 2015;372:1301-11.

4 Pandharipande PP, Girard TD, Jackson JC, et al. Long-term cognitive impairment after critical illness. N Engl J Med 2013;369:1306-16.

5 Young $\mathrm{P}$, Bailey M, Beasley R, et al. Effect of a buffered crystalloid solution vs saline on acute kidney injury among patients in the intensive care unit: The split randomized clinical trial. JAMA 2015;314:1701-10.
6 Perner A, Haase N, Guttormsen AB, et al. Hydroxyethyl starch 130/0.42 versus Ringer's acetate in severe sepsis. N Engl J Med 2012;367:124-34.

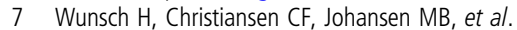
Psychiatric diagnoses and psychoactive medication use among nonsurgical critically ill patients receiving mechanical ventilation. JAMA 2014;311:1133-42.

8 Wunsch H, Guerra C, Barnato AE, et al. Three-year outcomes for Medicare beneficiaries who survive intensive care. JAMA 2010;303:849-56.

9 Garland A, Olafson K, Ramsey CD, et al. Distinct determinants of long-term and short-term survival in critical illness. Intensive Care Med 2014;40:1097-105.

10 Truwit JD, Bernard GR, Steingrub J, et al., National Heart, Lung, and Blood Institute ARDS Clinical Trials Network. Rosuvastatin for sepsis-associated acute respiratory distress syndrome. N Eng/ I Med 2014;370:2191-200.

11 Dinglas VD, Hopkins RO, Wozniak AW, et al. One-year outcomes of rosuvastatin versus placebo in sepsis-associated acute respiratory distress syndrome: prospective follow-up of SAlLS randomised trial. Thorax 2016;71:401-10.

12 Angus DC, Laterre PF, Helterbrand J, et al. The effect of drotrecogin alfa (activated) on long-term survival after severe sepsis. Crit Care Med 2004:32:2199-206.

13 Herridge MS, Cheung AM, Tansey CM, et al. One-year outcomes in survivors of the acute respiratory distress syndrome. N Eng/ I Med 2003;348:683-93.

14 Needham DM, Dinglas VD, Bienvenu OJ, et al. One year outcomes in patients with acute lung injury randomised to initial trophic or full enteral feeding: prospective follow-up of EDEN randomised trial. BMJ 2013;346:f1532.

15 van Beusekom I, Bakhshi-Raiez F, et al. Reported burden on informal caregivers of ICU survivors: a literature review. Crit Care 2016;20:16.

16 Williamson P, Altman D, Blazeby J, et al. Driving up the quality and relevance of research through the use of agreed core outcomes. J Health Serv Res Policy 2012;17:1-2.

17 Improving Long-Term Outcomes Research for Acute Respiratory Failure. http://www.improvelto.com/ (accessed 16 Mar 2016).

18 Iwashyna TJ, Cooke CR, Wunsch $\mathrm{H}$, et al. Population burden of long-term survivorship after severe sepsis in older Americans. J Am Geriatr Soc 2012;60:1070-7. 